\title{
NEITHER PUSH NOR PULL: STATE-FEEDBACK CONTROL FOR PRODUCTION SYSTEMS
}

\author{
Gašper Muši $\check{c ̆}^{(a)}$, Juliana Keiko Sagawa ${ }^{(b)}$ \\ (a)University of Ljubljana, Faculty of Electrical Engineering, Tržaška 25, 1000, Ljubljana, Slovenia \\ (b)Federal University of São Carlos, Production Engineering Department, Rodovia Washington Luís, Km 235, 13565 - \\ 905, São Carlos - SP, Brazil \\ (a)gasper.music@,fe.uni-lj.si, ${ }^{(b)}$ juliana@dep.ufscar.br
}

\begin{abstract}
Operations management techniques can benefit from integration of control theory methods when dealing with production and supply chain networks dynamics. In this context, we revisit a bond-graph based mathematical model that is able to capture the dynamics of multiworkstation production systems, and propose a state feedback control design to maintain work in process at desired levels. The closed loop performance of this real case inspired model was explored and simulations with the introduction of a disturbance in the production system were carried out. The proposed control design results in a disturbance-oriented behaviour that has advantages over pure push or pull systems commonly used in Production Planning and Control (PPC). In the given case, the results revealed that the model can provide prescriptive capacity adjustments and can help to define appropriate reference levels for the work in process in dynamic production environments.
\end{abstract}

Keywords: state feedback control; production planning and control; operations management

\section{INTRODUCTION}

Supply chain and production managers are faced with increased decision-making complexity, caused, among others, by the large variety of offered products, wide network of customers and suppliers, distributed production facilities, and customer demands on reduced delivery times.

From the operations perspective, it is known that the delivery performance is related to work in process (WIP) and throughput time (Lödding 2013). The trade-off between throughput rate and throughput time, and the direct relation of both with the WIP must be incorporated in any reasonable model of manufacturing systems (Lefeber 2012).

Various modelling approaches were proposed to cope with this trade-off in the context of production dynamics. The application of control theory principles, using feedback loops, control laws and transfer functions is one of the alternatives for this purpose (Duffie and Falu 2002). Another less explored option for dynamic modelling of production systems is the use of bond graphs (Ferney 2000).

An advantage of the bond graph methodology is its modular structure, i.e., once a set of graphs and elements is defined to represent basic entities (such as a machine or a buffer), several of these elements may be linked together into a more complex model to form different configurations. This makes the methodology attractive to be used by production managers and practitioners.

Adaptations of the bond graph methodology for modelling production systems (Ferney 2000) result in formal models that do not comply with the standard control theoretic synthesis methods. Thus, the design of good controllers for these models presents a substantial challenge. Hence, this paper aims to revisit a bond graph model of a multiproduct production system (Sagawa, Nagano, and Speranza Neto 2017) and presents a solution for the design of controllers for this system.

\section{BOND GRAPH MODELLING OF PRODUCTION SYSTEM}

The model of production system used in this paper was presented in Sagawa and Nagano (2015a,b), Sagawa, Nagano, and Speranza Neto (2017). The model depicts the dynamics of multi-product manufacturing system and was developed based on bond graph methodology. It is based on analogies between the manufacturing entities and electrical components, and involves the approximation of discrete systems as continuous systems (Ferney 2000). In terms of analogies, the machines are represented as resistors while the intermediate buffers are characterised as capacitors. The well-known expressions from electrical circuits are applied to form equations of these elements. A single workstation in this model comprises a machine and its precedent buffer, and is obtained from the combination of a capacitor, a resistor and a source of effort, as proposed by Ferney (2000).

In order to represent a multi-workstation system, Sagawa, Nagano, and Speranza Neto (2017) employ the concept of a material flow matrix (MFM) or probability flow matrix (PFM). When normalized, this matrix expresses the percentage of work content that flows from a workstation of a job shop to another one. In addition, 
ideal electrical transformers are introduced in the bond graph model to represent divergent junctions, where the output flow of a given workstation is split up into $k$ different inputs to downstream workstations.

This approach was applied to an 11-workstation system of a textile company with complex material flow (Sagawa and Nagano 2015). The production process starts with the extrusion of polymer to produce polypropylene thread, and continues with weaving in circular looms. After that, the various products go through different finishing operations, such as lamination, printing, cutting and stitching, according to the required customizations. The system may be classified as a unidirectional job shop or a general flow shop, since the products have different routings where operations might be skipped, but there are no reentryorder-flows.

The related model is described in detail in Sagawa, Nagano, and Speranza Neto (2017). Here, only the main equations and resulting matrix model are presented. The input to machine $i$ comes from a subset $S$ of machines upstream machine $i$, whose outputs feed machine $i$ to some extent. The rate of work in process (WIP) generation $\dot{q}_{i}$ on a given workstation is described by:

$\dot{q}_{i}(t)=\sum_{s \in S} \varphi_{i s} U_{S} \min \left(1, q_{s}(t)\right)-U_{i} \min \left(1, q_{i}(t)\right)$,

where $s \in S$ and $\varphi_{i s}$ may be defined as the non-zero elements of a characteristic material flow matrix $\varphi$ for the production system, out of its main diagonal. Parameter $U_{i}$ is associated to the processing frequency of a given machine, and $q_{i}$ is production volume stored in the machine preceding buffer. The min function is related to the approximation of discrete systems as continuous systems (Ferney 2000).

For the system under analysis, the matrix $\varphi$ is shown in Eq. (2).

$\boldsymbol{\varphi}=$
$\left[\begin{array}{ccccccccccc}-1 & 0 & 0 & 0 & 0 & 0 & 0 & 0 & 0 & 0 & 0 \\ 1 & -1 & 0 & 0 & 0 & 0 & 0 & 0 & 0 & 0 & 0 \\ 0 & 0,4300 & -1 & 0 & 0 & 0 & 0 & 0 & 0 & 0 & 0 \\ 0 & 0,2508 & 0,5118 & -1 & 0 & 0 & 0 & 0 & 0 & 0 & 0 \\ 0 & 0,1538 & 0,3140 & 0 & -1 & 0 & 0 & 0 & 0 & 0 & 0 \\ 0 & 0,0170 & 0,0347 & 0 & 0 & -1 & 0 & 0 & 0 & 0 & 0 \\ 0 & 0,0175 & 0,0020 & 0,2811 & 0,2811 & 0 & -1 & 0 & 0 & 0 & 0 \\ 0 & 0,0087 & 0,0010 & 0,1399 & 0,1399 & 0 & 0 & -1 & 0 & 0 & 0 \\ 0 & 0,0161 & 0,0018 & 0,2588 & 0,2588 & 0 & 0 & 0 & -1 & 0 & 0 \\ 0 & 0,0200 & 0,0023 & 0,3204 & 0,3204 & 0 & 0 & 0 & 0 & -1 & 0 \\ 0 & 0 & 0 & 0 & 0 & 0 & 0,2407 & 0,2407 & 0,2407 & 0,2407 & -1\end{array}\right]$

(2)

Some elements of this flow matrix directly correspond to the percentages of work content flowing from a workstation of a job shop to another one. For the cases where the input flow of a given machine is ruled by a combination of convergent and divergent junctions, then the coefficients of the flow matrix are appropriate products of these percentages (for details, see Sagawa, Nagano, and Speranza Neto (2017)). The matrix is lower triangular since there are no re-entry flows.

The only exception for the application of Eq. (1) regards to the first machine of the system (the extruder), whose input flow comes from the source of raw material, $U_{01}$. Adjusting Eq. (1) to it yields:

$\dot{q}_{1}(t)=U_{01}-U_{1} \min \left(1, q_{1}(t)\right)$.

Using the flow matrix $\varphi$, Eqs. (1) and (3) may be rewritten in matrix form, yielding the state model of the system:

$\dot{\mathbf{q}}(t)=\boldsymbol{\varphi} \cdot \operatorname{diag}(\mathbf{U}(t)) \cdot \min (1, \mathbf{q}(t))+\mathbf{0}_{1} U_{01}(t)$,

where system state $\mathbf{q}(t)$ is a $11 \times 1$ a vector of momentary production volumes stored in the system buffers, $\dot{\mathbf{q}}(t)$ is the corresponding vector of volume rates, $\varphi$ is material flow matrix defined by Eq. (2), and $\mathbf{U}(t)$ is a column vector of workstation processing frequencies. Expression $\operatorname{diag}(\mathbf{U}(t))$ denotes a $11 \times 11$ diagonal matrix whose nonzero elements are the elements of vector $\mathbf{U}(t), U_{01}(t)$ is the processing frequency of the source of raw material that feeds the system, and $\mathbf{0}_{1}$ is a $11 \times 1$ vector filled with zeros except at the first position, which contains $1\left(\mathbf{0}_{1}=\right.$ $\left.\left[\begin{array}{lllll}1 & 0 & 0 & \ldots & 0\end{array}\right]^{\mathrm{T}}\right)$. This state space model was used as the basis for the controller design proposed in the sequel.

\section{CONTROL DESIGN}

The main goal of the presented approach is WIP control, and WIP in the system corresponds to the material in the buffers of the stations. The related amounts of WIP in the buffers are the controlled variables of the system and the processing frequencies of the stations are the manipulated variables with additional manipulated input representing the source of material flow. Reference levels for the buffers are set so that there is enough material to process (to prevent starvation) but also the throughput times remain within acceptable range. The level of the buffers is monitored and the implemented controller performs capacity adjustments (i.e. it regulates the processing frequencies of the stations) in order to keep the material in the buffers at desired levels. To some extent, input control (order release) and output control (capacity control) are performed simultaneously, since input flow $U_{01}$ is also adjusted by the controller.

\subsection{Model linearization}

The standard state space controller design methods require a linear model. As the derived production model is nonlinear, it has to be adjusted.

First, the nonlinear expressions $\min \left(1, q_{j}\right)$ are omitted. In this way, the model only correctly describes the operation in the high production volume regime, which should be taken into account when setting the reference values for the buffer levels.

The corresponding linearized model is

$\dot{\mathbf{q}}(t)=\boldsymbol{\varphi} \cdot \mathbf{U}(t)+\mathbf{0}_{1} U_{01}(t)$,

The workstation processing frequency $U_{i}$ can be expressed as

$U_{i}=U_{i p}\left(1+u_{i}\right)$ 
where $U_{i p}$ denotes the steady state processing frequency of machine $i$, and $u_{i}$ is the relative processing frequency deviation from the steady state. A similar transformation is applied to the material input flow $U_{01}$. The deviation $u_{i}$ will represent a control input in the linearized model. The formulation (6) emphasises the fact that controlleradjusted processing frequencies will vary around the nominal steady state processing frequencies. These are calculated based on the average demand for each product family, and thus define the steady-state operation regime, which will be able to meet the demand for each product family.

The model (5) is further simplified by introducing an extended vector of processing frequencies $\mathbf{U}^{\mathbf{1}}=$ $\left[U_{1}, \ldots, U_{11}, U_{01}\right]^{T}$. Similarly, the diagonal matrix of processing frequencies is extended to $\operatorname{diag}\left(\mathbf{U}^{\mathbf{1}}\right)$. Taking (6) into account, the processing frequencies are expressed in vector form as

$\mathbf{U}^{\mathbf{1}}=\mathbf{U}_{\mathbf{p}}^{\mathbf{1}}+\operatorname{diag}\left(\mathbf{U}_{\mathbf{p}}^{\mathbf{1}}\right) \cdot \mathbf{u}_{\mathbf{f}}$.

It should be noted here that $\mathbf{U}_{\mathbf{p}}^{\mathbf{1}}$ is determined by the steady state relation $\boldsymbol{\varphi} \cdot \mathbf{U}_{\mathbf{p}}+U_{01 p} \mathbf{0}_{1}=\left[\boldsymbol{\varphi}, \mathbf{0}_{1}\right] \cdot \mathbf{U}_{\mathbf{p}}^{\mathbf{1}}=$ $\mathbf{0}$ and has a unique solution for a given $U_{01 p}$.

Considering (7) and the steady state relation, the model (5) is rewritten as

$\dot{\mathbf{q}}(t)=\left[\boldsymbol{\varphi}, \mathbf{0}_{1}\right] \cdot \operatorname{diag}\left(\mathbf{U}_{\mathbf{p}}^{\mathbf{1}}\right) \cdot \mathbf{u}_{\mathbf{f}}(t)$

which can be interpreted as a linear state space model with zero state matrix $\mathbf{A}=0$ and input matrix $\mathbf{B}$, such that

$\mathbf{B}=\left[\boldsymbol{\varphi}, \mathbf{0}_{1}\right] \cdot \operatorname{diag}\left(\mathbf{U}_{\mathbf{p}}^{\mathbf{1}}\right)$

\subsection{Control synthesis}

The basic full state feedback control law for the given case is

$\mathbf{u}_{f}=-\mathbf{K} \cdot \mathbf{q}$,

where $\mathbf{K}$ is a matrix of $(n+1) \times n$ feedback gains, $n$ representing the number of workstations.

As the goal of the controller is to maintain reference levels for the buffers, i.e., reference tracking, the state feedback control has to be modified to settle at nonzero $q$ values. This can be achieved by standard technique of adding integral action in state feedback control (Franklin, Powell, and Emami-Naeini 1994). To this end, the state vector $\mathbf{q}$ is augmented with additional $n$ state variables $q_{j I}$. These will maintain the desired operating point by acting as internal states of an integral controller. The integral action is implemented as a part of the full state feedback control law for the augmented system. The added state variables are related to deviation from the reference levels:

$q_{j I}^{\prime}=-e_{j}=q_{j}-r_{j}$ where $e_{j}$ is the control error, and $r_{j}$ is the reference value for buffer level $q_{j}$. Negative integral gain is assumed to comply with the negative sign in the state feedback equation.

For the case with zero state matrix, $\mathbf{A}=0$, the augmented system model is

$\left[\begin{array}{c}\dot{\mathbf{q}}_{I} \\ \dot{\mathbf{q}}\end{array}\right]=\left[\begin{array}{cc}0 & \mathbf{I}_{n} \\ 0 & 0\end{array}\right] \cdot\left[\begin{array}{c}\mathbf{q}_{I} \\ \mathbf{q}\end{array}\right]+\left[\begin{array}{l}0 \\ \mathbf{B}\end{array}\right] \mathbf{u}_{f}-\left[\begin{array}{c}\mathbf{I}_{n} \\ 0\end{array}\right] \mathbf{r}$,

and the corresponding control law is

$\mathbf{u}_{f}=-\left[\begin{array}{ll}\mathbf{K}_{I} & \mathbf{K}\end{array}\right]\left[\begin{array}{l}\mathbf{q}_{I} \\ \mathbf{q}\end{array}\right]$.

Note that the closed loop dynamics is determined by augmented system matrices $\mathbf{A}_{a}=\left[\begin{array}{cc}0 & \mathbf{I}_{n} \\ 0 & 0\end{array}\right], \mathbf{B}_{a}=\left[\begin{array}{l}0 \\ \mathbf{B}\end{array}\right]$, $\mathbf{C}_{a}=\left[\begin{array}{ll}0 & \mathbf{I}_{n}\end{array}\right], \mathbf{D}_{a}=0$, and the extended feedback gain matrix $\mathbf{K}_{a}=\left[\begin{array}{ll}\mathbf{K}_{I} & \mathbf{K}\end{array}\right]$.

The variations in the processing frequencies imposed by the controller represent in fact capacity adjustments, which cannot be performed in a continuous manner. In practice, a corresponding control action is applied in a discrete manner, by means of overtime or extra shifts, for instance. In order to better represent that, the continuous processing frequency adjustments provided by the controller should be converted into discrete adjustments in specified time intervals, such as one day, five days or ten days.

Conversion to discrete-time control adjustments is straightforward. The continuous state space model (8) has to be discretized to

$\mathbf{q}(k+1)=\mathbf{A}_{d} \mathbf{q}(k)+\mathbf{B}_{d} \mathbf{u}_{f}(k)$,

with a chosen sampling time $T_{s}, t=k T_{s}$. Sampling time should be relatively short in comparison to the expected system settling time.

When the control inputs are piecewise constant over the sample time $T_{s}$, matrices $\mathbf{A}_{d}$ and $\mathbf{B}_{d}$ can be expressed as $\mathbf{A}_{d}=e^{\mathbf{A} T_{s}}, \mathbf{B}_{d}=\int_{0}^{T_{s}} e^{\mathbf{A} T_{s}} \mathbf{B} d t$. For the system under analysis, $\mathbf{A}=0$, therefore $\mathbf{A}_{d}=\mathbf{I}_{n}$ (with $n$ equal to the number of workstations) and $\mathbf{B}_{d}=\mathbf{B} T_{s}$. As in the continuous case, $\mathbf{C}_{d}=\mathbf{I}_{n}$ and $\mathbf{D}_{d}=0$. Similarly to (10), the augmented model for the reference tracking is composed as

$\left[\begin{array}{l}\mathbf{q}_{I}(k+1) \\ \mathbf{q}(k+1)\end{array}\right]=\mathbf{A}_{a d} \cdot\left[\begin{array}{l}\mathbf{q}_{I}(k) \\ \mathbf{q}(k)\end{array}\right]+\mathbf{B}_{a d} \mathbf{u}_{f}(k)-\left[\begin{array}{c}\mathbf{I}_{n} \\ 0\end{array}\right] \mathbf{r}(k)$,

where $\mathbf{A}_{a d}=\left[\begin{array}{cc}\mathbf{I}_{n} & \mathbf{I}_{n} \\ 0 & \mathbf{I}_{n}\end{array}\right], \mathbf{B}_{a d}=\left[\begin{array}{c}0 \\ \mathbf{B}_{d}\end{array}\right]$ and, additionally, $\mathbf{C}_{a d}=\left[\begin{array}{ll}0 & \mathbf{I}_{n}\end{array}\right], \mathbf{D}_{a d}=0$.

Then, the specific values of the gain matrix for a given case can be derived by various synthesis methods.

In the following, the control matrix $\mathrm{K}$ can be derived by a discrete-time version of LQR method, which minimizes the quadratic cost function 


$$
J\left(\mathbf{u}_{f}\right)=\sum_{k=1}^{\infty}\left(\mathbf{q}(k)^{T} \mathbf{Q q}(k)+\mathbf{u}_{f}(k)^{T} \mathbf{R} \mathbf{u}_{f}(k)+\right.
$$$$
\left.2 \mathbf{q}(k)^{T} \mathbf{N u}_{f}(k)\right) \text {. }
$$

Matrices $\mathbf{Q}, \mathbf{R}$ and $\mathbf{N}$ in (14) weigh the contribution of various terms to the cost function value. The $\mathbf{Q}$ matrix determines the relative importance of different combinations of the product of states, $\mathbf{R}$ defines the contribution of terms related to manipulated variables (control input), and $\mathbf{N}$ determines the importance of mixed terms.

Commonly, only squared states and squared inputs are taken into account, meaning that the $\mathbf{Q}$ and $\mathbf{R}$ matrices become diagonal, and $\mathbf{N}=0$. Appropriate coefficient ratios are chosen to compensate for differences in the signal magnitudes. A well-known rule of thumb (Bryson's Rule, see Franklin, Powell, and Emami-Naeini 1994) is to weight signal $s_{i}$ by $\omega_{i}^{2} /\left(s_{i}\right)_{\max }^{2}$ with $\sum_{i} \omega_{i}=1$ for signals related to $\mathbf{Q}$ and $\mathbf{R}$, respectively. Weights $\omega_{i}$ add additional relative weighting on the various components of the state/control vector. Additionally, the balance between the influence of state deviations and the influence of control effort to the cost function is achieved by scaling matrix $\mathbf{R}$ with constant $\rho$. In the investigated case, the state vector was scaled based on reference buffer levels, and the same scaling was used for both integrator states and buffer levels. No additional relative weighting was used, and we have no additional information that could be used for control signal scaling, therefore:

$$
\mathbf{Q}=\left[\begin{array}{cc}
\operatorname{diag}\left(1 / r_{i}^{2}\right) & 0 \\
0 & \operatorname{diag}\left(1 / r_{i}^{2}\right)
\end{array}\right], \quad \mathbf{R}=\rho \cdot \mathbf{I}_{n u}, \quad \mathbf{N}=0
$$

The relative weight between state and control vector components was set to $\rho=100$.

\section{SIMULATION RESULTS}

The closed loop control system with state feedback was initially simulated under a scenario, where all buffers are initially empty, and the desired reference levels are set to 0.1 times the benchmark levels adopted in Sagawa and Nagano (2015). Sampling period of 1 day was chosen, which corresponds to daily changes of the prescribed capacity levels. Three months of production are simulated.

A partial breakdown of station 2 occurs after a month of operation, i.e. at time 30 . The station consists of 12 parallel looms, and the partial breakdown corresponds to the malfunction of one of these looms. The repair time was artificially extended to 20 days before the looms are set back into operation, in order to have a clearer picture of the system's response to such a disturbance. The corresponding controller signal is shown in Fig. 1.

The controller response shows that the workstations 1,2 and 3 start to operate with processing frequencies above the respective steady state frequencies, in order to fill the initially empty buffers. Simultaneously, the stations downstream station 3 (i.e., station 4 to station 11) process material with a frequency below the steady state frequency until there is enough WIP in the buffers to be processed (see U4 to U11 at Fig. 1). After circa 20 days, the system reaches steady state with the WIP at the reference levels (the relative errors close to zero correspond to relative processing frequencies close to 1 in Fig. 1).

It can be observed that controller responds to a disturbance (partial failure of the station, i.e. breakdown of one of the looms on day 30), by gradually increasing the capacity of the remaining functioning part to approximately $111 \%$ in the first few days after the disturbance, and then decreasing it to $108 \%$ until the machine recovery, to keep the WIP at required levels. After the recovery, the capacity is set back to normal, after a few days of transient adjustments.

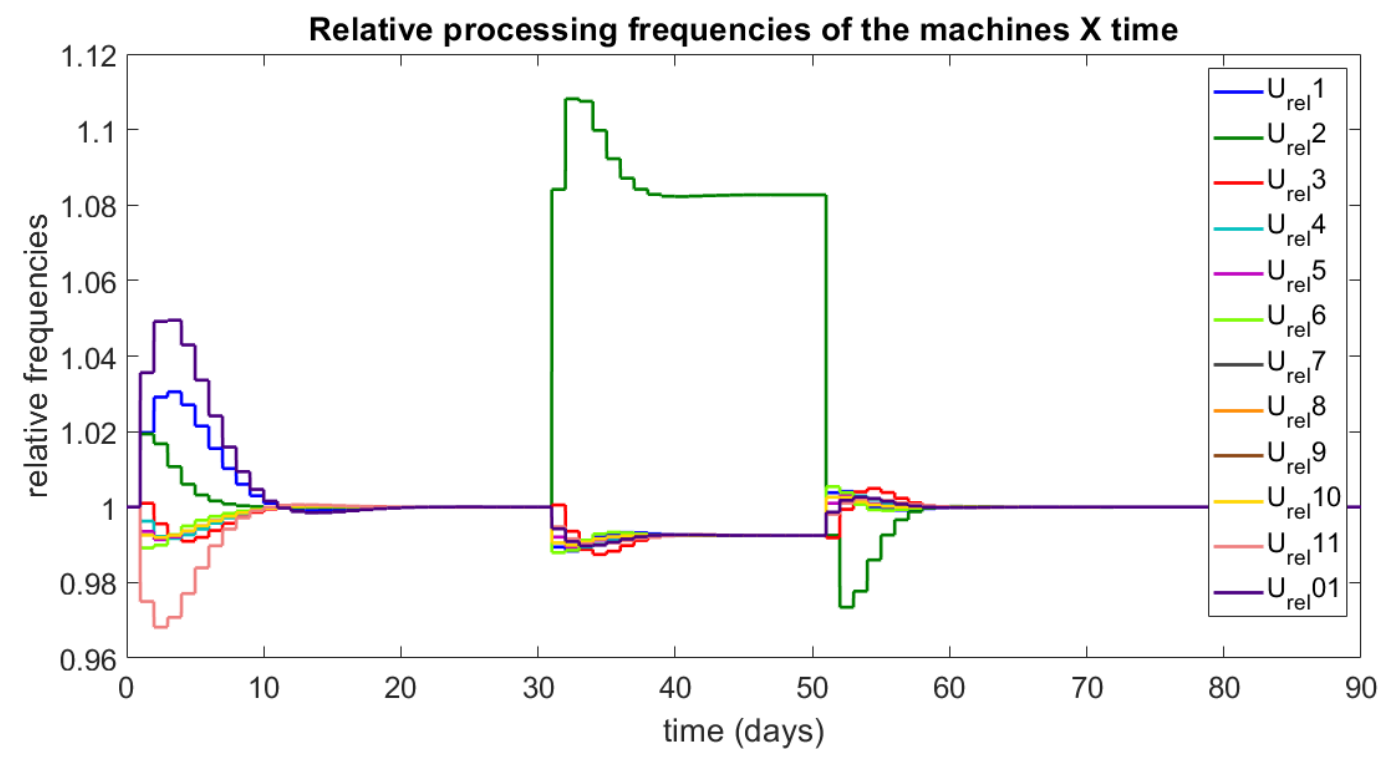

Figure 1: Discretization of the controller signal for 1 day intervals (i.e. recommended capacity adjustments for the stations) 


\subsection{Testing scenarios}

A set of testing scenarios were defined in order to test the ability of the proposed production control strategy to respond to disturbances. Simulations with the synthesised discrete-time controller were carried out under the following scenarios:

1. The same scenario used in the simulation already presented, but with reference levels set as 0.2 and 0.05 times the benchmark levels (to verify the impact of the variation of the reference buffer levels);

2. A scenario with reference levels set as 0.2 times the benchmark levels and the breakdown of station 8 from day 30 to day 32;

3. A scenario with reference levels set as 0.2 times the benchmark levels and the breakdown of station 4 from day 30 to day 32 .
The results are shown in: Fig. 2-3, for the first scenario; Fig. 4-5, for the second scenario; Fig. 6-7, for the third scenario.

One remark regarding Fig. $5 \mathrm{~b}$ and 7 is that the processing frequencies of the broken stations 4 and 8 from day 30 to 32 shown in the figures correspond to how the controller would intend to react, but not to the actual processing frequency of the broken station. In fact, the processing frequencies of these stations during the breakdown are kept at zero.

The tests with scenario 1 showed that, with too low reference levels for the WIP, some buffers become empty (this corresponds to relative errors equal to -1, as in Fig. $3 \mathrm{~b})$. This means that the local work in process is not sufficient to absorb the disturbance occurred, and some machines starve. Buffers work as a safety "cushion" against the interruption of production flow, and the simulations with the proposed model may help to define adequate reference levels for this cushion.

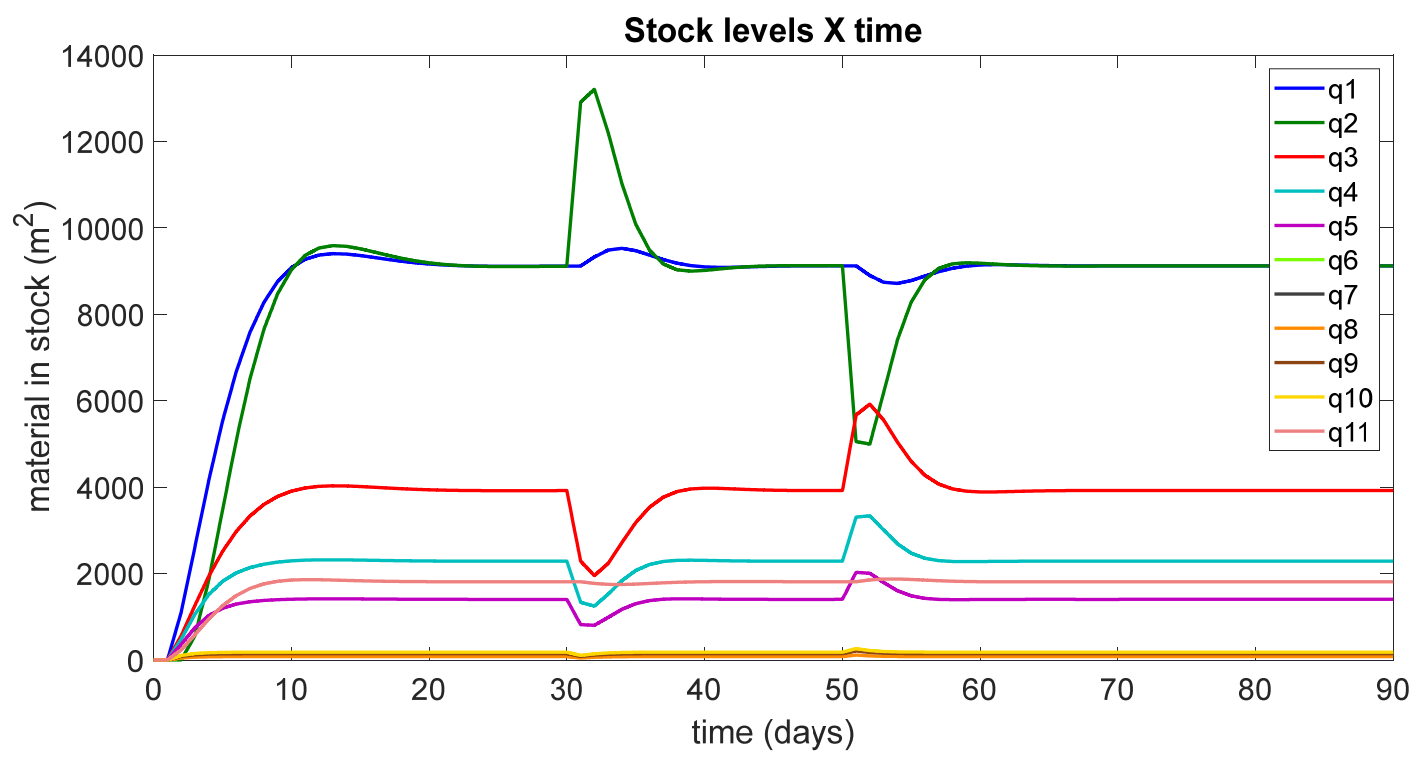

Figure 2: Simulation results for scenario 1 (discrete-time LQR), with reference levels equal to $0.2 \mathrm{x}$ the benchmark levels and partial breakdown of the looms (station 2) - stock levels
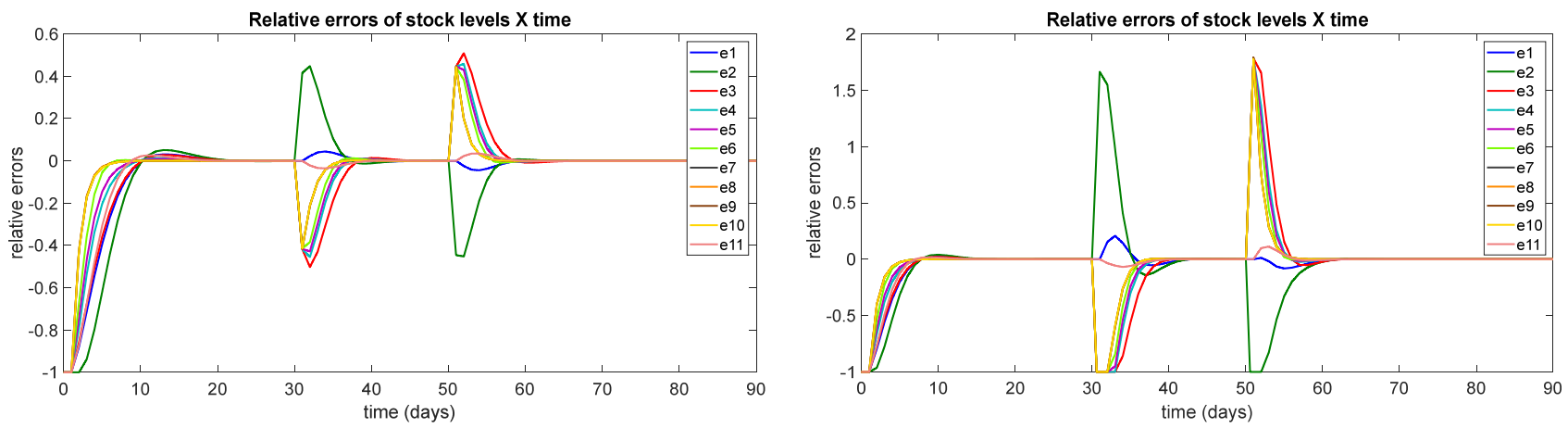

Figure 3 - Simulation results for scenario 1 (discrete-time LQR) - relative errors in the stock levels: a) with reference levels as 0.2 times benchmark values; b) with reference levels as 0.05 times benchmark values. 

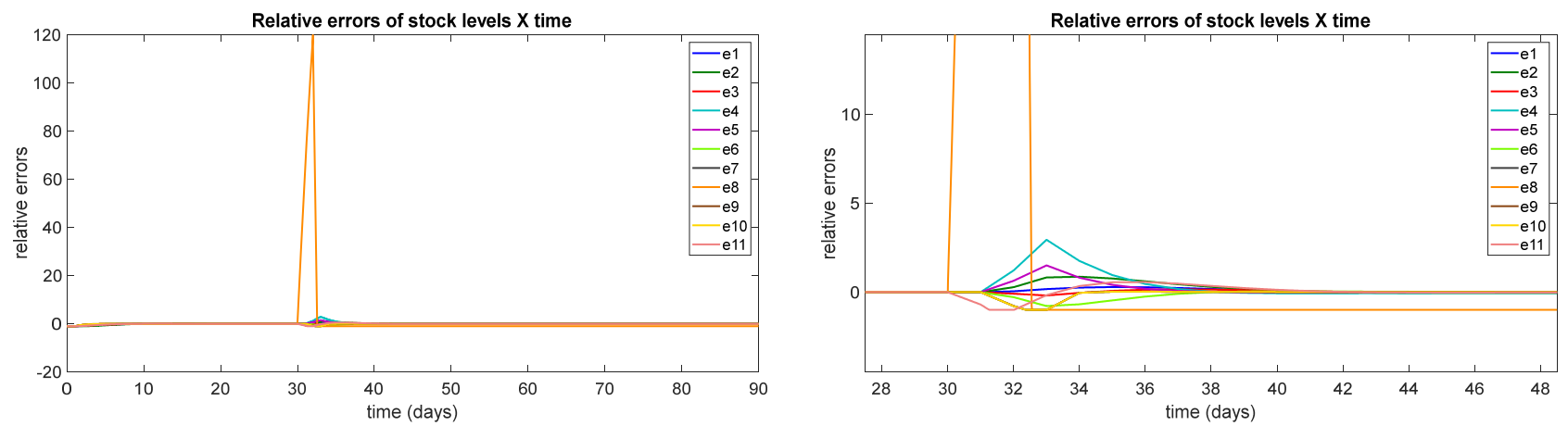

Figure 4 - Simulation results for scenario 2 (discrete-time LQR) - relative errors in the stock levels: a) overview; b) view of the detail
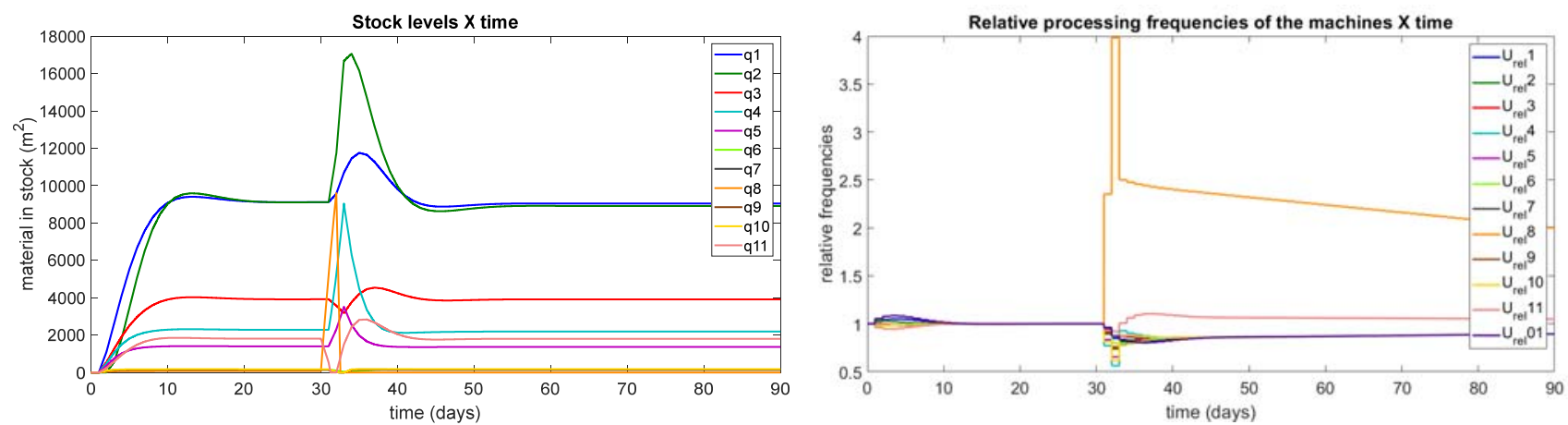

Figure 5 - Simulation results for scenario 2: a) stock levels; b) relative processing frequencies

In scenario 2, the breakdown of machine 8 causes a temporary increase of WIP around $9500 \mathrm{~m}^{2}$ of material ( $q_{8}$ in Fig. 5a). This peak of seems to be very pronounced in relative terms (Fig. 4a) because the reference level for buffer 8 is significantly lower than the levels of other buffers. This level was defined as a function of the total volume of material that is processed in each station. Station 8 is parallel to 3 other stations with similar function (stations 7, 9 and 10) and performs a finishing operation. As the products of four families do not require this finishing, it processes a relatively low volume of material. After 3 days, when the station 8 has already recovered, the material is totally consumed and the buffer becomes empty. The significant increase in the processing frequency of station 8 (Fig. 5b) represents how the controller would intend to react (in fact, the processing frequency of the station is kept at zero during days 30 to 32). After the disturbance, the station 8 should work 2.5 times more (Fig. 5b). The remaining stations decrease their rate of operation during the breakdown. With this reaction, after the disturbance, the buffer levels tend to get back to the reference levels.

In Fig. 6b, depicting scenario 3, it is possible to see that the starvation of the stations $7,8,9$ and 10 occurs just during the breakdown, i.e. during 2 days (curves are superposed). As soon as machine 4 restarts to operate with 1.4 times its capacity, then the downstream buffers start to be filled again; after an overshoot, they stabilize close to the desired levels. It takes longer for the buffer of machine 4 to reach its desired level, but it doesn't mean that the production is interrupted: it just means that all its production is feeding the downstream machines, in order to keep delivering the desired output flows.

The controller decreases the processing frequencies of the stations upstream station 4 in order not to aggravate the build-up of WIP at station 4 (Fig. 7). On the other hand, the processing frequency of the stations immediately downstream (stations 5 and 6) is also decreased to respond to a lower incoming flow from machine 4. This shows that some stations react looking downstream (i.e. looking at the customer process, as in a pull system) and some stations react looking upstream (i.e. looking at the supplier process). This means that the system is neither pulled nor pushed; the control is centralized and rather focused on responding to the disturbance.

\section{DISCUSSION}

In the push system, the scheduling/sequencing of tasks in each station is defined according to a centralized strategy and based on exogenous information. The stations keep producing independently of the situation downstream in the process. In the pull systems, on the contrary, the material and information flows are in the opposite directions, i.e. the consumption of the downstream stations determines the amount and sequence of production of a given station. Thus, in the pull system, this reaction is based on endogenous information and is 

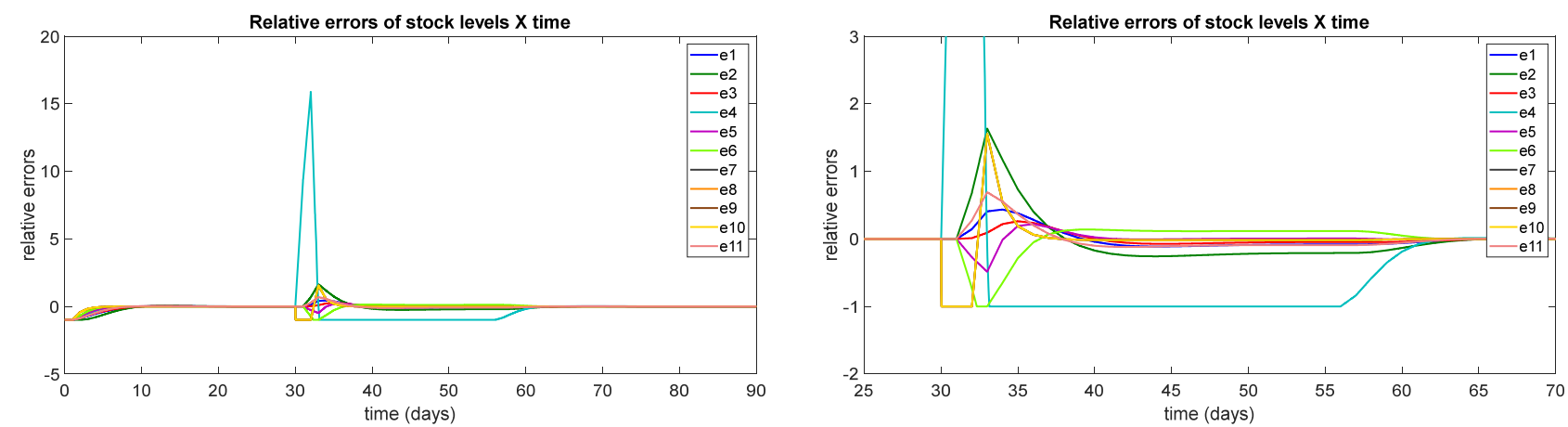

Figure 6: Simulation results for scenario 3 (discrete-time LQR) - relative errors in the stock levels: a) overview; b) detail

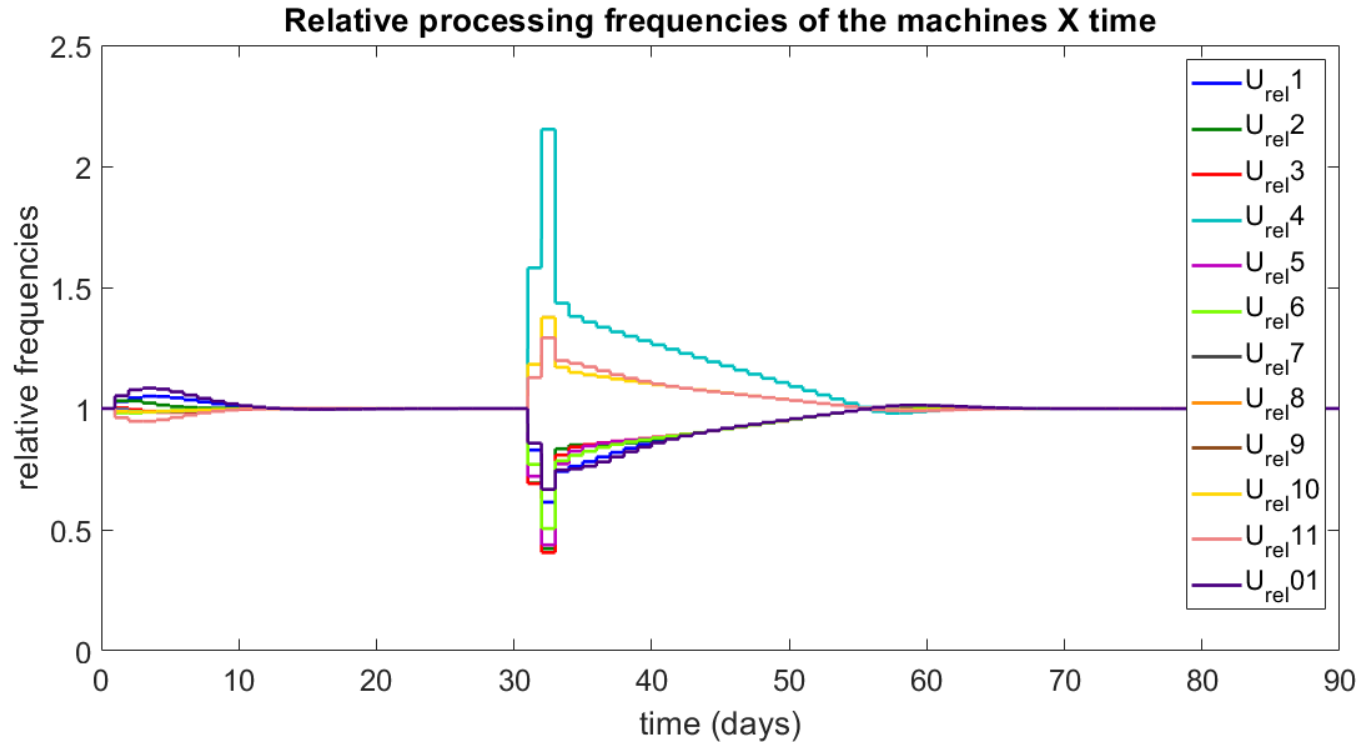

Figure 7: Simulation results for scenario 3 (discrete-time LQR) - relative processing frequencies

local, i.e. driven by the immediately subsequent station. As a result, a pull production system explicitly limits the amount of WIP that can reside in the system (Hopp and Spearman 2004).

In previous studies (Sagawa and Nagano, 2015; Sagawa, Nagano, and Speranza Neto 2017), feedback control was implemented to simulate a pull system, where the controller of each station just reacted based on the error of the buffer downstream, i.e. the buffer of the next station. This local approach based on proportional (P) controllers proved not to be so effective, since a high overshoot (i.e. surplus of material) in some buffers was observed. When the system was subjected to machine breakdowns, significant peaks were observed, even when a local Proportional Integral (PI) controller was used (Sagawa and Freitag 2016).

In the proposed model, instead of being local, the control is centralized, in the sense that a holistic view of the system is considered in order to define the reaction of each station. As observed in the simulation of scenario 3, if the disturbance occurs in the middle of the system, some stations react by looking upstream and some stations react by looking downstream; in fact, they react to the disturbance itself. This is an alternative operation mode that can be more effective in some situations than a pure "push" or "pull" system, see also Sagawa and Mušič (2019).

\section{CONCLUSIONS}

This paper presented a production control strategy for a dynamic multi-product production system, and the synthesis of the corresponding production controller that improves the system's operation. This approach based on state feedback control design techniques is novel in the sense that it had not been applied in prior studies concerning bond graph models that depict the shop floor of a manufacturing system.

The choice of the modelling framework and the application of control synthesis techniques open the way to use the simulation of production systems in a prescriptive rather than descriptive manner. The controllers of the workstations suggest necessary capacity adjustments in order to achieve specific goals, e.g. to keep the WIP under control, even in the presence of disturbances. Other relevant goals might be set, such as attending fluctuating demand levels, for instance. 
One of the possible directions to be explored in future works is to investigate the combination of advanced control techniques with discrete-event simulation.

\section{ACKNOWLEDGMENTS}

The authors acknowledge the financial support from the Slovenian Research Agency (research core funding No. P2-0219).

\section{REFERENCES}

Duffie N., Falu, I., 2002. Control-Theoretic Analysis of a Closed-Loop PPC System. CIRP Annals Manufacturing Technology, 51 (1), 379-382. doi: 10.1016/s0007-8506(07)61541-8.

Ferney M., 2000. Modelling and Controlling product manufacturing systems using bond-graphs and state equations: continuous systems and discrete systems which can be represented by continuous models. Production Planning \& Control, 11 (1), 7-19.

Franklin G.F., Powell, J.D., Emami-Naeini, A., 1994). Feedback control of dynamic systems (3rd ed.). Reading, MA: Addison-Wesley.

Hopp, W.J., Spearman, M.L., 2004. To pull or not to pull: what is the question? Manufacturing \& service operations management, 6 (2), 133-148.

Lefeber, E., 2012. Modeling and Control of Manufacturing Systems. In: Armbruster, D., Kempf, K.G. (Eds.). Decision Policies for Production Networks, Springer-Verlag London, 930. doi: 10.1007/978-0-85729-644-3_2

Lödding, H., 2013. Handbook of Manufacturing Control: Fundamentals, Description, Configuration. Springer-Verlag Berlin Heidelberg.

Sagawa, J.K., Nagano, M.S., 2015a. Applying bond graphs for modelling the manufacturing dynamics. IFAC-PapersOnLine 48 (3), 2047-2052.

Sagawa, J.K., Nagano, M.S., 2015b. Modeling the dynamics of a multi-product manufacturing system: A real case application. European Journal of Operational Research, 244 (2), 624-636. doi: 10.1016/j.ejor.2015.01.017.

Sagawa, J.K., Freitag, M., 2016. A simulation model for the closed-loop control of a multi-workstation production system. Proceedings of the 9th EUROSIM Congress on Modelling and Simulation, Oulu. Linköping: Linköping University Press.

Sagawa, J.K., Nagano, M.S., Speranza Neto, M., 2017. A closed-loop model of a multi-station and multiproduct manufacturing system using bond graphs and hybrid controllers. European Journal of Operational Research, 258 (2), 677-691. doi: 10.1016/j.ejor.2016.08.056.

Sagawa, J.K., Mušič, G., 2019. Towards the use of bond graphs for manufacturing control: Design of controllers, International Journal of Production Economics, 214, 53-72. doi: 10.1016/j.ijpe.2019. 03.017 .

\section{AUTHORS BIOGRAPHY}

JULIANA KEIKO SAGAWA received B.Sc., M.Sc. and $\mathrm{Ph} . \mathrm{D}$. degrees in production engineering from the University of São Paulo, Brazil in 2006, 2008, and 2013, respectively. She is Adjunct Professor at the Faculty of Production Engineering of the Federal University of São Carlos. Her research interests include dynamic modelling of production systems, planning, scheduling and some organizational topics such as integration and servitization. More information can be found at: http: //lattes. cnpq. br/6549481623896786.

GAŠPER MUŠIČ received B.Sc., M.Sc. and Ph.D. degrees in electrical engineering from the University of Ljubljana, Slovenia in 1992, 1995, and 1998, respectively. He is Full Professor at the Faculty of Electrical Engineering, University of Ljubljana. His research interests are in discrete event and hybrid dynamical systems, supervisory control, planning, scheduling, and industrial informatics. His Web page can be found at http://msc.fe.uni-lj.si/ Staff.asp. 